Martin Dindoš, University of North Carolina Chapel Hill, Phillips Hall CB

\#3250, Chapel Hill NC 27599. e-mail: dindos@math.unc.edu

\title{
ON SERIES WITH ALTERNATING SIGNS IN THE EUCLIDEAN METRIC
}

\begin{abstract}
This paper presents one of many interesting aspects of relatively convergent series. Namely, given a sequence of elements of a Hilbert space we consider all possible ways of placing plus or minus signs in front of these elements to create an alternating sequence. In a convenient metric it makes sense to ask what is the 'size' of the set of those choices of + or - for which the resulting series converges. The term 'size' here refers to either Baire category or Lebesgue measure of this set. It turns out that especially the question of the Lebesgue measure of this set is quite intriguing and leads to interesting results generalizing known results for real-valued sequence.
\end{abstract}

\section{Introduction}

Relatively convergent series are studied in many monographs and articles. As we mentioned in the abstract we study the convergence of the series

$$
\sum_{n=1}^{\infty}(-1)^{a_{n}} b_{n}
$$

where $\left(a_{n}\right)_{n \in \mathbb{N}}$ is a sequence of zeros and ones and $\left(b_{n}\right)_{n \in \mathbb{N}}$ is a sequence of elements of a real ${ }^{1}$ Hilbert space $H$. The inner product on $H$ will be denoted by $\langle.,$.$\rangle .$

Key Words: relatively convergent series, Baire category, Lebesgue measure, Euclidean metric, $\ell^{2}$ theorem

Mathematical Reviews subject classification: Primary 28A21, secondary 40A05, 54E52, $28 \mathrm{~A} 03$

Received by the editors March 29, 1999

${ }^{1}$ The fact that $H$ is a real Hilbert space is not particularly important, since any complex Hilbert space of dimension $n$ can be also viewed as a real Hilbert space of dimension $2 n$ with modified inner product. 
For a fixed sequence $\left(b_{n}\right)_{n \in \mathbb{N}}$ we want to consider the sets

$$
\begin{aligned}
& \mathcal{C}=\left\{\left(a_{n}\right)_{n \in \mathbb{N}} \in\{0,1\}^{N} ; \text { the series (1) converges }\right\} \text { and } \\
& \mathcal{B}=\left\{\left(a_{n}\right)_{n \in \mathbb{N}} \in\{0,1\}^{N} ; \exists M>0 \forall k\left|\sum_{n=1}^{k}(-1)^{a_{n}} b_{n}\right| \leq M\right\} .
\end{aligned}
$$

The set $\mathcal{C}$ contains all sequences $\left(a_{n}\right)_{n \in \mathbb{N}}$ for which series (1) converges and the set $\mathcal{B}$ contains sequences $\left(a_{n}\right)_{n \in \mathbb{N}}$ for which the series (1) has bounded partial sums. Apparently, $\mathcal{C} \subset \mathcal{B}$. The question of what these sets are is definitely nontrivial only if

$$
\text { there is }\left(a_{n}\right)_{n \in \mathbb{N}} \in\{0,1\}^{N} \text { such that } \sum_{n=1}^{\infty}(-1)^{a_{n}} b_{n} \text { diverges, }
$$

because otherwise the series (2) is convergent for any choice of $\left(a_{n}\right)_{n \in \mathbb{N}}$. (Later we will formulate a sufficient condition on $\left(b_{n}\right)_{n \in \mathbb{N}}$ that guarantees $\left.(2)\right)$.

Denote by $|$.$| the norm of the space H$ defined by $|x|=\langle x, x\rangle^{\frac{1}{2}}$ for $x \in H$. Since we are working in the space $\{0,1\}^{\mathbb{N}}$ there are several choices of metrics we can consider. Namely, we can equip this space either with the Frèchet or Baire metric. In this paper we would like to consider yet another metric which is closely connected with standard Euclidean metric on the real line. This will allow us to study not only categorical 'size' of the sets $\mathcal{C}$ and $\mathcal{B}$ but also their Lebesgue measure.

Consider therefore the map $\varphi:\{0,1\}^{\mathbb{N}} \rightarrow[0,1]$ defined as follows.

$$
\varphi\left(\left(a_{n}\right)_{n \in \mathbb{N}}\right)=\sum_{n=1}^{\infty} \frac{a_{n}}{2^{n}} \text { for }\left(a_{n}\right)_{n \in \mathbb{N}} \in\{0,1\}^{\mathbb{N}} .
$$

This is definitely a surjective map but not bijective because the sequences

$$
\begin{aligned}
& \left(a_{1}, a_{2}, . ., a_{n}, 0,1,1,1, \ldots\right) \\
& \left(a_{1}, a_{2}, . ., a_{n}, 1,0,0,0, \ldots\right)
\end{aligned}
$$

are mapped onto the same point.

Now we have two options. Either we define $d_{E}(a, b)=|\varphi(a)-\varphi(b)|$, which is a pseudometric on the space $\{0,1\}^{\mathbb{N}}$ or we can make this space a little smaller by dropping all sequences of the type $\left(a_{1}, a_{2}, . ., a_{n}, 0,1,1,1, \ldots\right)$ or $\left(a_{1}, a_{2}, \ldots, a_{n}, 1,0,0,0, \ldots\right)$. Let for example take

$$
\mathcal{M}=\{0,1\}^{\mathbb{N}} \backslash\left\{\left(a_{1}, a_{2}, \ldots, a_{n}, 0,1,1,1, \ldots\right) ; a_{i} \in\{0,1\} i=1,2, \ldots, n\right\} .
$$


Then $\left(\mathcal{M}, d_{E}\right)$ is a complete metric space. All the material that follows work for both options and therefore we will not emphasize explicitly which particular space we have in mind. We rather formulate all theorems for $\left(\{0,1\}^{\mathbb{N}}, d_{E}\right)$ keeping in mind that it also works for $\left(\mathcal{M}, d_{E}\right)$ and will call $d_{E}$ the Euclidean metric (slightly abusing standard terminology).

A lot about the set $\mathcal{C}$ is known in case $H=\mathbb{R}$. Namely, we know that for sequences satisfying (2) this set is of first Baire category in $\left(\{0,1\}^{\mathbb{N}}, d_{E}\right)$ and moreover there is a beautiful theorem about the Lebesgue measure of the set $\varphi(\mathcal{C})$.

$\ell^{2}$ theorem. The Lebesgue measure of the set $\varphi(\mathcal{C})$ is one if and only if $\sum_{n=1}^{\infty}\left|b_{n}\right|^{2}<\infty$, i.e., $\left(b_{1}, b_{2}, \ldots\right) \in \ell^{2}$. Otherwise this set has Lebesgue measure zero.

This theorem is a consequence of Kolmogorov three series theorem (see e.g. $[\mathrm{Du}]$ for a reference). Our aim will be to generalize this theorem for any Hilbert space $H$ where we mainly have in mind infinite dimensional spaces since for $\mathbb{R}^{n}$ the extension of the $\ell^{2}$ theorem is trivial using the theorem itself on each coordinate.

This work has also been motivated by very interesting results that have appeared in works of V. László and T. Šalát. They studied the series

$$
\sum_{n=1}^{\infty} a_{n} b_{n}
$$

where $\sum_{n=1}^{\infty} b_{n}$ is a divergent real nonnegative series and $\left(a_{n}\right)_{n \in \mathbb{N}}$ is a sequence of zeros and ones. According to their work in $[\mathrm{L}-\check{S}$ ] and $[\check{S}]$ the set of sequences $\left(a_{n}\right)_{n \in \mathbb{N}}$ for which the series (3) converges (i.e. $\mathcal{C}$ ) is of the first Baire category on $\left(\{0,1\}^{\mathbb{N}}, d_{E}\right)$ and the Lebesgue measure of $\varphi(\mathcal{C})$ is zero.

\section{Categorical Size of the Set of Convergent Sequences}

In this section we present the discussion of the sets $\mathcal{B}$ and $\mathcal{C}$ defined above in terms of their Baire category in the complete metric space $\left(\{0,1\}^{\mathbb{N}}, d_{E}\right)$. Before proving the main result of this section we need to do some preparatory work.

Let $\mathcal{D}$ be the set of all sequences $\left(a_{n}\right)_{n \in \mathbb{N}} \in\{0,1\}^{\mathbb{N}}$ that correspond to dyadic rationals, i.e.,

$$
\mathcal{D}=\left\{\left(a_{n}\right)_{n \in \mathbb{N}} \in\{0,1\}^{\mathbb{N}} ; \varphi\left(\left(a_{n}\right)_{n \in \mathbb{N}}\right) \text { is a dyadic rational }\right\} .
$$


Lemma 2.1. Consider the complete metric space $\left(\{0,1\}^{\mathbb{N}}, d_{E}\right)$. Let $k$ be a positive integer, $c$ a real number and $\left(b_{1}, b_{2}, \ldots b_{k}\right)$ a finite sequence of elements of $H$. Let $\mathcal{S}$ be the set

$$
\mathcal{S}=\left\{\left(a_{n}\right)_{n \in \mathbb{N}} \in\{0,1\}^{\mathbb{N}} ;\left|(-1)^{a_{1}} b_{1}+(-1)^{a_{2}} b_{2}+\cdots+(-1)^{a_{k}} b_{k}\right|>c\right\} .
$$

Then there exists a countable set $E \subset \mathcal{D}$ such that the set $\mathcal{S} \backslash E$ is an open subset of the space $\left(\{0,1\}^{\mathbb{N}}, d_{E}\right)$.

Proof. It suffices to show the following claim.

$$
a=\left(a_{n}\right)_{n \in \mathbb{N}} \in \mathcal{S} \backslash \mathcal{D} \Longrightarrow a \in \operatorname{int} \mathcal{S}
$$

Once we show this, then clearly the exceptional set $E=\mathcal{S} \backslash$ int $\mathcal{S} \subset \mathcal{D}$ is countable.

Consider therefore a sequence $\left(a_{n}\right)_{n \in \mathbb{N}} \in \mathcal{S} \backslash \mathcal{D}$. Denote by $\underline{a}$ and $\bar{a}$ two real numbers with the following decimal dyadic expansion.

$$
\begin{aligned}
& \underline{a}=0, a_{1} a_{2} \ldots a_{k} 000 \cdots=\sum_{i=1}^{k} \frac{a_{i}}{2^{i}} \\
& \bar{a}=0, a_{1} a_{2} \ldots a_{k} 111 \cdots=\sum_{i=1}^{k} \frac{a_{i}}{2^{i}}+\frac{1}{2^{k}} .
\end{aligned}
$$

(For clarity of our notation we used a decimal comma instead of a dot). The assumption $\left(a_{n}\right)_{n \in \mathbb{N}} \notin \mathcal{D}$ guarantees that $\underline{a}<\varphi\left(\left(a_{n}\right)_{n \in \mathbb{N}}\right)<\bar{a}$. We put

$$
\varepsilon=\min \left\{\varphi\left(\left(a_{n}\right)_{n \in \mathbb{N}}\right)-\underline{a}, \bar{a}-\varphi\left(\left(a_{n}\right)_{n \in \mathbb{N}}\right)\right\}>0 .
$$

Now any sequence $\left(c_{n}\right)_{n \in \mathbb{N}}$ whose distance from $\left(a_{n}\right)_{n \in \mathbb{N}}$ is less that $\varepsilon$ must have first $k$ elements identical with $\left(a_{n}\right)_{n \in \mathbb{N}}$ and therefore such $\left(c_{n}\right)_{n \in \mathbb{N}}$ is in $\mathcal{S}$. This means that the sequence $\left(a_{n}\right)_{n \in \mathbb{N}}$ belongs to int $\mathcal{S}$.

Now we are ready to prove that the both $\mathcal{B}$ and $\mathcal{C}$ are sets of first Baire category in the complete metric space $\left(\{0,1\}^{\mathbb{N}}, d_{E}\right)$ provided (2) holds.

Theorem 2.2. Consider the series

$$
\sum_{n=1}^{\infty}(-1)^{a_{n}} b_{n}
$$

where $\left(b_{n}\right)_{n \in \mathbb{N}}$ is a given sequence of elements of $H$. Assume that the condition (2) holds, namely

$$
\text { there is }\left(a_{n}\right)_{n \in \mathbb{N}} \in\{0,1\}^{\mathbb{N}} \text { such that } \sum_{n=1}^{\infty}(-1)^{a_{n}} b_{n} \text { diverges. }
$$


Then

$$
\mathcal{C}=\left\{\left(a_{n}\right)_{n \in \mathbb{N}} \in\{0,1\}^{\mathbb{N}} ; \text { the series }(1) \text { converges }\right\}
$$

as well as,

$$
\mathcal{B}=\left\{\left(a_{n}\right)_{n \in \mathbb{N}} \in\{0,1\}^{\mathbb{N}} ; \exists M>0 \forall k\left|\sum_{n=1}^{k}(-1)^{a_{n}} b_{n}\right| \leq M\right\}
$$

are sets of first Baire category in the complete space $\left(\{0,1\}^{\mathbb{N}}, d_{E}\right)$.

Proof. Since $\mathcal{C} \subset \mathcal{B}$, proving the theorem for the set $\mathcal{B}$ will suffice. $\mathcal{B}$ can be written as

$$
\mathcal{B}=\bigcup_{M=1}^{\infty} \bigcap_{k=1}^{\infty}\left\{\left(a_{n}\right)_{n \in \mathbb{N}} ;\left|\sum_{n=1}^{k}(-1)^{a_{n}} b_{n}\right| \leq M\right\}
$$

Let $F_{i}$ be

$$
F_{i}=\bigcap_{k=1}^{\infty}\left\{\left(a_{n}\right)_{n \in \mathbb{N}} ;\left|\sum_{n=1}^{k}(-1)^{a_{n}} b_{n}\right| \leq i\right\} .
$$

We claim that the closure of $F_{i}$ is nowhere dense. From this our theorem follows. To establish this claim we should look more closely at the sets of the form

$$
\left\{\left(a_{n}\right)_{n \in \mathbb{N}} ;\left|\sum_{n=1}^{k}(-1)^{a_{n}} b_{n}\right| \leq M\right\}
$$

The complement of such a set is a set of the form $\mathcal{S}$ from the Lemma 2.1. Consequently this lemma gives us that for each $i \in \mathbb{N}$ there is a countable set $E_{i} \subset \mathcal{D}$ such that the closure of $F_{i}$ is equal to $\overline{F_{i}}=F_{i} \cup E_{i}$. Now we are ready to prove that each $\overline{F_{i}}$ is nowhere dense. Take any $\left(a_{n}\right)_{n \in \mathbb{N}} \in \overline{F_{i}}$. We want to show that in any $\varepsilon$ neighborhood of this sequence there is a sequence $\left(c_{n}\right)_{n \in \mathbb{N}}$ which does not belong to $\overline{F_{i}}$. Let $\left(d_{n}\right)_{n \in \mathbb{N}} \in\{0,1\}^{\mathbb{N}}$ be a sequence for which $\sum_{n=1}^{\infty}(-1)^{d_{n}} b_{n}$ diverges. Existence of such sequence follows from (2). If we modify this sequence and denote the modified sequence again $\left(d_{n}\right)_{n \in \mathbb{N}}$ we can achieve that

$$
\lim _{k \rightarrow \infty}\left|\sum_{n=1}^{k}(-1)^{d_{n}} b_{n}\right|=\infty .
$$

If necessary, a further modification of the sequence $\left(d_{n}\right)_{n \in \mathbb{N}}$ can guarantee that $\left(d_{n}\right)_{n \in \mathbb{N}} \notin \mathcal{D}$.

Define $\left(c_{n}\right)_{n \in \mathbb{N}}$ by

$$
c_{n}= \begin{cases}a_{n}, & \text { for } n=1,2, \ldots, k \\ d_{n}, & \text { otherwise }\end{cases}
$$


Here $k$ is chosen big enough to assure that the distance between $\left(a_{n}\right)_{n \in \mathbb{N}}$ and $\left(c_{n}\right)_{n \in \mathbb{N}}$ is less that given $\varepsilon$. Namely, we take $k$ to be a positive integer such that $\frac{1}{2^{k}}<\varepsilon$.

It is clear now that the sequence $\left(c_{n}\right)_{n \in \mathbb{N}}$ does not belong to $F_{i}$ since

$$
\sum_{n=k+1}^{\infty}(-1)^{c_{n}} b_{n}=\sum_{n=k+1}^{\infty}(-1)^{d_{n}} b_{n},
$$

and according to (4) the sum on the right is unbounded in the norm of $H$. Also since $\left(d_{n}\right)_{n \in \mathbb{N}} \notin \mathcal{D}$ we have that $\left(c_{n}\right)_{n \in \mathbb{N}} \notin E_{i}$. These two things together imply that $\left(c_{n}\right)_{n \in \mathbb{N}}$ does not belong to $\overline{F_{i}}$. This finishes the proof.

Remark. Close analysis of the $F_{i}$ gives that it is a $G_{\delta}$ set. Therefore $\mathcal{B}$ is a Borel set (in fact $G_{\delta \sigma}$ ). Similarly the set $\mathcal{C}$ is also Borel $\left(G_{\delta \sigma \delta}\right)$ since

$$
\mathcal{C}=\bigcap_{m=1}^{\infty} \bigcup_{K=1}^{\infty} \bigcap_{k=K}^{\infty} \bigcap_{l=1}^{\infty}\left\{\left(a_{n}\right)_{n \in \mathbb{N}} ;\left|\sum_{n=k}^{k+l}(-1)^{a_{n}} b_{n}\right| \leq \frac{1}{m}\right\} .
$$

It is also interesting to ask whether the above result about the sets $\mathcal{B}, \mathcal{C}$ can be strengthened by replacing first Baire category by porosity. We postpone the discussion of this problem until we develop more material on the Lebesgue measure of the sets $\varphi(\mathcal{B})$ and $\varphi(\mathcal{C})$.

\section{Lebesgue Measure}

As we indicated before, since the Euclidean metric $d_{E}$ on the space $\{0,1\}^{\mathbb{N}}$ is defined via the map $\varphi:\{0,1\}^{\mathbb{N}} \rightarrow[0,1]$ given by

$$
\varphi\left(\left(a_{n}\right)_{n \in \mathbb{N}}\right)=\sum_{n=1}^{\infty} \frac{a_{n}}{2^{n}} \text { for }\left(a_{n}\right)_{n \in \mathbb{N}} \in\{0,1\}^{\mathbb{N}},
$$

it makes good sense to ask what is the Lebesgue measure of the images of the sets $\mathcal{C}$ and $\mathcal{B}$ under the map $\varphi$. Said differently we want to compute the numbers $\lambda(\varphi(\mathcal{C}))$ and $\lambda(\varphi(\mathcal{B}))$.

First we state a simple result which gives us that the measure of each of these sets is either zero or one (i.e., the full measure on the interval $[0,1]$ ). In case $H=\mathbb{R}$ this is the famous Kolmogorov $0-1$ law. Here we closely follow the paper [ک̌S].

Definition 3.1. A measurable set $E \subset(0,1)$ (Here we can also admit the closed interval $[0,1]$.) is called homogenous if there exists a number $d \in[0,1]$ such that for any interval $I \subset(0,1)$ we have $\frac{\lambda(I \cap E)}{\lambda(I)}=d$. 
As a consequence of the Lebesgue theorem we have the following result.

Proposition 3.2. If $E$ is homogenous, then the number $d$ in Definition 3.1 is either 0 or 1 ; i.e., $\lambda(E)=0$ or $\lambda(E)=1$.

Proposition 3.3. Let $Q$ be a dense subset of the real line $\mathbb{R}$. Let $E$ be $a$ measurable subset of $(0,1)$. If for any interval $I \subset(0,1)$ and any $q \in Q$ for which

$$
q+I=\{q+i ; i \in I\} \subset(0,1)
$$

we have

$$
\lambda(I \cap E)=\lambda((q+I) \cap E),
$$

then the set $E$ is homogenous, i.e., $\lambda(E)=0$ or $\lambda(E)=1$.

Proof. If $\lambda(E)=0$ the proposition holds. Assume therefore that $\lambda(E)>0$. According to the Lebesgue theorem there exists a point $z \in(0,1)$ for which we have

$$
\lim _{I \rightarrow z} \frac{\lambda(I \cap E)}{\lambda(I)}=1 .
$$

Here $I \rightarrow z$ means the limit over any sequence of intervals $I$ containing $z$ whose diameter goes to zero.

Pick any $x \in(0,1)$. We show that $\lim _{I \rightarrow x} \frac{\lambda(I \cap E)}{\lambda(I)}=1$. From this our proposition follows immediately, because then each point of the interval $(0,1)$ is a Lebesgue density point of $E$.

It is sufficient to show that given any sequence of intervals $\left(I_{n}\right)_{n \in \mathbb{N}}$ for which $I_{n} \rightarrow x$ we have $\lim _{n \rightarrow \infty} \frac{\lambda\left(I_{n} \cap E\right)}{\lambda\left(I_{n}\right)}=1$. Obviously, for each $n \in \mathbb{N}$ there is a number $q_{n} \in Q$ such that the interval $J_{n}=q_{n}+I_{n}$ contains $z$. This also means that $J_{n} \rightarrow z$ and by (5) we then have

$$
\lim _{n \rightarrow \infty} \frac{\lambda\left(I_{n} \cap E\right)}{\lambda\left(I_{n}\right)}=\lim _{n \rightarrow \infty} \frac{\lambda\left(J_{n} \cap E\right)}{\lambda\left(J_{n}\right)}=1,
$$

so the proposition is established.

Corollary 3.4. The sets $\varphi(\mathcal{C})$ and $\varphi(\mathcal{B})$ are homogenous. Therefore their measure is either 0 or 1 .

Proof. Clearly both sets are Lebesgue measurable. This follows from the previous section where we have shown that $\mathcal{B}$ is a $G_{\delta \sigma}$ and $\mathcal{C}$ is a $G_{\delta \sigma \delta}$ set in $\left(\{0,1\}^{\mathbb{N}}, d_{E}\right)$. Same argument shows that $\varphi(\mathcal{B})$ and $\varphi(\mathcal{C})$ are of the corresponding types on $[0,1]$. 
Let $Q=\left\{\frac{k}{2^{n}} ; k, n \in \mathbb{N}\right\}$. According to the previous theorem it suffices to show that for any $I \subset(0,1)$ and any $q \in Q$ for which $q+I \subset(0,1)$ we have

$$
\lambda(I \cap E)=\lambda((q+I) \cap E) .
$$

(Here $E$ stands for $\varphi(\mathcal{C})$ and $\varphi(\mathcal{B})$, respectively.) However, (6) is quite obvious. If we take any $x \in E$ its dyadic expansion is $\varphi^{-1}(x)=\left\{a_{1}, a_{2}, a_{3}, \ldots\right\}$ where $a_{n} \in\{0,1\}$. By adding any number $q \in Q$ whose dyadic expansion has tail $\{\ldots, 0,0,0, \ldots\}$ to $x$ we alter the first $k$ digits of the dyadic expansion of $x$ but the tails of dyadic expansions of $x$ and $x+q$ are the same; i.e.,

$$
\varphi^{-1}(x+q)=\left\{b_{1}, b_{2}, \ldots, b_{k}, a_{k+1}, a_{k+2}, \ldots\right\} .
$$

This means that

$$
\begin{aligned}
& x \in \varphi(\mathcal{C}) \Longleftrightarrow x+q \in \varphi(\mathcal{C}), \\
& x \in \varphi(\mathcal{B}) \Longleftrightarrow x+q \in \varphi(\mathcal{B}) .
\end{aligned}
$$

because the convergence (boundedness) of the series (1) depends only on the tail of the dyadic expansion of $x$ and $x+q$ respectively. Thus we have

$$
\begin{aligned}
& I \cap \varphi(\mathcal{C})=(q+I) \cap \varphi(\mathcal{C}) \\
& I \cap \varphi(\mathcal{B})=(q+I) \cap \varphi(\mathcal{B}) .
\end{aligned}
$$

This result gives us that the sets $\varphi(\mathcal{C}), \varphi(\mathcal{B})$ have measure zero or one, but it is not obvious which option applies. As we will see later there are sequences $\left(b_{n}\right)_{n \in \mathbb{N}}$ for which $\lambda(\varphi(\mathcal{C}))=\lambda(\varphi(\mathcal{B}))=1$, and there are others for which $\lambda(\varphi(\mathcal{C}))=\lambda(\varphi(\mathcal{B}))=0$. If fact we get the necessary and sufficient condition extending the $\ell^{2}$ theorem. It will also follows that there is no sequence $\left(b_{n}\right)_{n \in \mathbb{N}}$ for which $\lambda(\varphi(\mathcal{C}))=0$ but $\lambda(\varphi(\mathcal{B}))=1$.

The rest of this section requires some use of probability methods. Therefore in all that follows our probability space will be the interval $[0,1)$ with probability measure $P=\lambda$ the Lebesgue measure on $[0,1)$.

First we introduce our notation. Let $x \in[0,1)$. Then $x=\sum_{n=1}^{\infty} \frac{a_{n}(x)}{2^{n}}$ is the dyadic expansion of the number $x$. (To avoid the ambiguity in case of a dyadic rational we always in such situation take the terminal dyadic expansion of number $x$; i.e., with tail $\{\ldots, 0,0,0, \ldots\})$. Then each $n=1,2, \ldots$

$$
a_{n}:[0,1) \rightarrow\{0,1\}
$$

is a real function, i.e., a random variable. Moreover we have

$$
\varphi\left(\left\{a_{1}(x), a_{2}(x), a_{3}(x), \ldots\right\}\right)=x \text { for all } x \in[0,1) .
$$

It is a simple exercise to show the following. 
Proposition 3.5. The random variables $a_{1}(x), a_{2}(x), a_{3}(x), \ldots$ are independent. This means that for any integer $n \in \mathbb{N}$ and any $n$-tuple $k_{1}, k_{2}, \ldots, k_{n}$ we have

$$
P\left(a_{1}(x)=k_{1}, a_{2}(x)=k_{2}, \ldots, a_{n}(x)=k_{n}\right)=\prod_{i=1}^{n} P\left(a_{i}(x)=k_{i}\right) .
$$

For any $n \in \mathbb{N}$ define $S_{n}:[0,1) \rightarrow H$ by $S_{n}(x)=\sum_{i=1}^{n}(-1)^{a_{i}(x)} b_{i}$. The reason the Kolmogorov result does not extend immediately to our case is that the function $S_{n}$ is no longer a random variable since it takes values in $H$. Nevertheless $\left|S_{n}\right|^{2}=\left\langle S_{n}, S_{n}\right\rangle$ still takes values is $\mathbb{R}$; i.e., it is a random variable. This leads to the following lemma.

Lemma 3.6. If $\varepsilon>0$, then $P\left(\max _{1 \leq i \leq n}\left|S_{i}\right| \geq \varepsilon\right) \leq \frac{1}{\varepsilon^{2}} \sum_{i=1}^{n}\left|b_{i}\right|^{2}$ for $n=$ $1,2,3, \ldots$

Proof. Denote by $A_{i}$ the sets

$$
A_{i}=\left\{x \in[0,1) ;\left|S_{i}\right| \geq \varepsilon \text { but }\left|S_{j}\right|<\varepsilon \text { for } j<i\right\} .
$$

Apparently, $A_{i}$ are disjoint and

$$
\begin{aligned}
E\left(\left|S_{n}\right|^{2}\right)= & \int_{0}^{1}\left\langle S_{n}(x), S_{n}(x)\right\rangle d x \geq \sum_{i=1}^{n} \int_{A_{i}}\left\langle S_{n}(x), S_{n}(x)\right\rangle d x \\
= & \sum_{i=1}^{n} \int_{A_{i}}\left\langle S_{i}(x), S_{i}(x)\right\rangle+2\left\langle S_{i}(x), S_{n}(x)-S_{i}(x)\right\rangle \\
& +\left\langle S_{n}(x)-S_{i}(x), S_{n}(x)-S_{i}(x)\right\rangle d x \\
\geq & \sum_{i=1}^{n} \int_{A_{i}}\left|S_{i}\right|^{2} d x+\sum_{i=1}^{n} \int_{0}^{1} 2\left\langle S_{i} \chi_{A_{i}}, S_{n}(x)-S_{i}(x)\right\rangle d x .
\end{aligned}
$$

In the last step we used the fact that $\left|S_{n}(x)-S_{k}(x)\right|^{2} \geq 0$. Here $\chi_{A_{i}}$ denotes the indicator function of the set $A_{i}$. Our claim is that the integral in the second sum is zero for any $i \in\{1,2, \ldots, n\}$. To see this we have to realize that $S_{i} \chi_{A_{i}}$ is only a function of the random variables $a_{1}, \ldots, a_{i}$ whereas $S_{n}(x)-S_{i}(x)$ is a function of the random variables $a_{i+1}, \ldots, a_{n}$. Naturally, these two sets of random variables are independent. Put

$$
\mathcal{D}_{i_{1}, i_{2}, \ldots, i_{n}}=\left\{x \in[0,1) ; a_{k}(x)=i_{k} \text { for } k=1,2, \ldots n\right\} \text { where } i_{k} \in\{0,1\} .
$$


Then the sets $\mathcal{D}_{i_{1}, i_{2}, \ldots, i_{n}}$ are disjoint, they cover the interval $[0,1)$, each has measure $\frac{1}{2^{n}}$, and therefore the integral in the above sum can be written as

$$
\begin{aligned}
\int_{0}^{1}\left\langle S_{k} \chi_{A_{k}}, S_{n}(x)\right. & \left.-S_{k}(x)\right\rangle d x \\
& =\frac{1}{2^{n}} \sum_{i_{1}, i_{2}, \ldots, i_{n} \in\{0,1\}}\left\langle f\left(i_{1}, \ldots, i_{k}\right), g\left(i_{k+1}, \ldots, i_{n}\right)\right\rangle,
\end{aligned}
$$

where

$$
\begin{aligned}
f\left(i_{1}, \ldots, i_{k}\right) & = \begin{cases}\sum_{j=1}^{k}(-1)^{i_{j}} b_{j} & \text { if }\left|\sum_{j=1}^{k}(-1)^{i_{j}} b_{j}\right| \geq \varepsilon \text { but } \\
0 & \left|\sum_{j=1}^{l}(-1)^{i_{j}} b_{j}\right|<\varepsilon \text { for } l<k\end{cases} \\
g\left(i_{k+1}, \ldots, i_{n}\right) & =\sum_{j=k+1}^{n}(-1)^{i_{j}} b_{j} .
\end{aligned}
$$

Now for any $i_{1}, i_{2}, \ldots, i_{n}$ if we let

$$
j_{l}= \begin{cases}1-i_{l} & \text { for } 1 \leq l \leq k \\ i_{l} & \text { otherwise }\end{cases}
$$

we can see that

$$
\left\langle f\left(i_{1}, \ldots, i_{k}\right), g\left(i_{k+1}, \ldots, i_{n}\right)\right\rangle+\left\langle f\left(j_{1}, \ldots, j_{k}\right), g\left(j_{k+1}, \ldots, j_{n}\right)\right\rangle=0 .
$$

Thus the terms in the sum (7) can be grouped into pairs whose sum is zero. Therefore the whole sum (7) has to be zero. If we use this and the fact that on each of the set $A_{i}$ we have $\left|S_{i}\right| \geq \varepsilon$ we get

$$
E\left(\left|S_{n}\right|^{2}\right) \geq \sum_{i=1}^{n} \int_{A_{i}}\left|S_{i}\right|^{2} d x \geq \sum_{i=1}^{n} \varepsilon^{2} P\left(A_{i}\right)=\varepsilon^{2} P\left(\max _{1 \leq i \leq n}\left|S_{i}\right| \geq \varepsilon\right) .
$$

Hence if we establish

$$
E\left(\left|S_{n}\right|^{2}\right)=\sum_{i=1}^{n}\left|b_{i}\right|^{2}
$$

we are done. To see this compute

$$
\left\langle S_{n}, S_{n}\right\rangle=\sum_{i=1}^{n}\left|b_{i}\right|^{2}+2 \sum_{1 \leq i<j \leq n}\left\langle(-1)^{a_{i}(x)} b_{i},(-1)^{a_{j}(x)} b_{j}\right\rangle .
$$


We need to show that the expected value of each term in the second sum is zero. This follows from the fact that $a_{i}$ and $a_{j}$ are independent random variables plus a similar argument to the one we have used for the second term in (7). This concludes our proof.

Define now

$$
K_{n}(x)=\sum_{1 \leq i<j \leq n}(-1)^{a_{i}(x)+a_{j}(x)}\left\langle b_{i}, b_{j}\right\rangle
$$

The following lemma, similar to the previous one, will be also needed.

Lemma 3.7. If $\varepsilon>0$, then

$$
P\left(\max _{1 \leq i \leq n}\left|K_{i}\right| \geq \varepsilon\right) \leq \frac{1}{\varepsilon^{2}} \sum_{1 \leq i<j \leq n}\left|\left\langle b_{i}, b_{j}\right\rangle\right|^{2}, \quad n=1,2,3, \ldots .
$$

Proof. We proceed as before.

$$
A_{i}=\left\{x \in[0,1) ;\left|K_{i}\right| \geq \varepsilon \text { but }\left|K_{j}\right|<\varepsilon \text { for } j<i\right\} .
$$

Apparently, the sets $A_{i}$ are disjoint and

$$
\begin{aligned}
E\left(K_{n}^{2}\right) & =\int_{0}^{1} K_{n}(x)^{2} d x \geq \sum_{i=1}^{n} \int_{A_{i}} K_{n}(x)^{2} d x \\
& =\sum_{i=1}^{n} \int_{A_{i}} K_{i}(x)^{2}+2 K_{i}(x)\left(K_{n}(x)-K_{i}(x)\right)+\left(K_{n}(x)-K_{i}(x)\right)^{2} d x \\
& \geq \sum_{i=1}^{n} \int_{A_{i}}\left|K_{i}\right|^{2} d x+\sum_{i=1}^{n} \int_{0}^{1} 2\left(K_{i} \chi_{A_{i}}\right)\left(K_{n}(x)-K_{i}(x)\right) d x .
\end{aligned}
$$

In the last step we used the fact that $\left(K_{n}(x)-K_{k}(x)\right)^{2} \geq 0$. Here $\chi_{A_{i}}$ as before means the indicator function of the set $A_{i}$. Our claim is that the integral in second sum is zero for any $i \in\{1,2, \ldots, n\}$. Consider the sets $\mathcal{D}_{i_{1}, i_{2}, \ldots, i_{n}}$ defined in previous lemma. The integral in the last sum can be written as

$$
\begin{aligned}
\int_{0}^{1}\left(K_{k} \chi_{A_{k}}\right)\left(K_{n}(x)-K_{k}(x)\right) d x & \\
= & \frac{1}{2^{n}} \sum_{i_{1}, i_{2}, \ldots, i_{n} \in\{0,1\}} f\left(i_{1}, \ldots, i_{k}\right) g\left(i_{1}, i_{2}, \ldots, i_{k}, i_{k+1}, \ldots, i_{n}\right),
\end{aligned}
$$


where

$$
\begin{aligned}
f\left(i_{1}, \ldots, i_{k}\right) & =\chi_{A_{k}}\left(\sum_{1 \leq j<l \leq k}(-1)^{i_{j}+i_{l}}\left\langle b_{j}, b_{l}\right\rangle\right) \\
g\left(i_{1}, \ldots, i_{k}, i_{k+1}, \ldots, i_{n}\right) & =\sum_{\substack{1 \leq j<l \leq n \\
k<l}}(-1)^{i_{j}+i_{l}}\left\langle b_{j}, b_{l}\right\rangle .
\end{aligned}
$$

What is troubling here is the dependence of the function $g$ on indices $i_{1}, \ldots, i_{k}$. Fortunately the situation is not that bad. If we fix $i_{1}, \ldots, i_{k}$ and run the above sum for all combination $i_{k+1}, \ldots, i_{n}$, we can observe that

$$
\begin{aligned}
& g\left(i_{1}, \ldots, i_{k}, i_{k+1}, \ldots, i_{n}\right)+g\left(i_{1}, \ldots, i_{k}, 1-i_{k+1}, \ldots, 1-i_{n}\right) \\
& =G\left(i_{k+1}, \ldots, i_{n}\right)+G\left(1-i_{k+1}, \ldots, 1-i_{n}\right)
\end{aligned}
$$

where $G$ is defined by $G\left(i_{k+1}, \ldots, i_{n}\right)=\sum_{k+1 \leq j<l \leq n}(-1)^{i_{j}+i_{l}}\left\langle b_{j}, b_{l}\right\rangle$. Thus we get

$\int_{0}^{1}\left(K_{k} \chi_{A_{k}}\right)\left(K_{n}(x)-K_{k}(x)\right) d x=\frac{1}{2^{n}} \sum_{i_{1}, i_{2}, \ldots, i_{n} \in\{0,1\}} f\left(i_{1}, \ldots, i_{k}\right) G\left(i_{k+1}, \ldots, i_{n}\right)$,

replacing small $g$ by capital $G$. This solves our problem because now we can write

$$
\begin{aligned}
\int_{0}^{1}\left(K_{k} \chi_{A_{k}}\right) & \left(K_{n}(x)-K_{k}(x)\right) d x \\
= & \frac{1}{2^{n}}\left(\sum_{i_{1}, \ldots, i_{k} \in\{0,1\}} f\left(i_{1}, \ldots, i_{k}\right)\right)\left(\sum_{i_{k+1}, \ldots, i_{n} \in\{0,1\}} G\left(i_{k+1}, \ldots, i_{n}\right)\right) .
\end{aligned}
$$

The final point finishing our argument is that clearly

$$
\sum_{i_{k+1}, \ldots, i_{n} \in\{0,1\}} G\left(i_{k+1}, \ldots, i_{n}\right)=0 .
$$

Thus our integral vanishes. If we substitute this result into (10) and the fact that on set $A_{i}\left|K_{i}\right| \geq \varepsilon$ we get

$$
E\left(K_{n}^{2}\right) \geq \sum_{i=1}^{n} \int_{A_{i}}\left|K_{i}\right|^{2} d x \geq \sum_{i=1}^{n} \varepsilon^{2} P\left(A_{i}\right)=\varepsilon^{2} P\left(\max _{1 \leq i \leq n}\left|K_{i}\right| \geq \varepsilon\right) .
$$


Hence if we establish

$$
E\left(K_{n}^{2}\right)=\sum_{1 \leq i<j \leq n}\left|\left\langle b_{i}, b_{j}\right\rangle\right|^{2}
$$

we are done. To see this compute

$$
K_{n}^{2}=\sum_{1 \leq i<j \leq n}\left|\left\langle b_{i}, b_{j}\right\rangle\right|^{2}+\sum_{\substack{1 \leq i<j \leq n \\ 1 \leq k<l \leq n \\(i, j) \neq(k, l)}}(-1)^{a_{i}+a_{j}+a_{l}+a_{k}}\left\langle b_{i}, b_{j}\right\rangle\left\langle b_{k}, b_{l}\right\rangle .
$$

We need to show that the expected value of the last term is zero. However, this can be proved using techniques that have already appeared several times in this paper.

Now we are ready to prove the main theorem of this section, the generalized $\ell^{2}$ theorem for any Hilbert space $H$. As we will see this proof does not use any "heavy artillery" of probability theory as is the case of the proof of the Kolmogorov three series theorem where a quite complicated variant of the central limit theorem is used. We will instead use the measure theory as our main tool.

Theorem 3.8. [Generalized $\ell^{2}$ theorem] Let $\left(b_{n}\right)_{n \in \mathbb{N}}$ be any sequence of elements of a Hilbert space $H$. Then the Lebesgue measure of the sets $\varphi(\mathcal{C})$ and $\varphi(\mathcal{B})$ is one if and only if

$$
\sum_{n=1}^{\infty}\left|b_{n}\right|^{2}<\infty
$$

If (12) does not hold these sets have Lebesgue measure zero.

As a corollary we get the following.

Corollary 3.9. Condition (2) holds if $\sum_{n=1}^{\infty}\left|b_{n}\right|^{2}=\infty$.

Remark. Further generalization of Theorem 3.8 by replacing the Hilbert space $H$ by a Banach space in the statement of the Theorem 3.8 fails. There are many examples (e.g., $\ell^{p}, p \neq 2$ ) where the analogous theorem for such spaces fails.

Proof of Theorem 3.8 We first prove that if (12) holds, then the Lebesgue measure of the set $\varphi(\mathcal{C})$ is one (and therefore same is true about $\varphi(\mathcal{B})$ ). The key is to use Lemma 3.6. According to it for any positive integers $M, N$ we have 


$$
P\left(\max _{M \leq i \leq N}\left|S_{i}-S_{M}\right| \geq \varepsilon\right) \leq \frac{1}{\varepsilon^{2}} \sum_{i=M+1}^{N}\left|b_{i}\right|^{2} .
$$

Letting $N \rightarrow \infty$ we get

$$
P\left(\max _{M \leq i}\left|S_{i}-S_{M}\right| \geq \varepsilon\right) \leq \frac{1}{\varepsilon^{2}} \sum_{i=M+1}^{\infty}\left|b_{i}\right|^{2} .
$$

Now we also let $M \rightarrow \infty$. The right side tends to zero and therefore if we denote by $w_{M}$ the number $w_{M}=\sup _{m, n \geq M}\left|S_{m}-S_{n}\right|$, we obtain that $w_{M} \rightarrow 0$ almost everywhere as $M \rightarrow \infty$ since

$$
P\left(w_{M} \geq 2 \varepsilon\right) \leq P\left(\max _{M \leq i}\left|S_{i}-S_{M}\right| \geq \varepsilon\right) \rightarrow 0 .
$$

This means the sequence $\left(S_{M}\right)_{M \in \mathbb{N}}$ of partial sums of the series (1) is Cauchy almost everywhere and thus convergent in our Hilbert space.

To establish the converse we have to do a little more work. Assume that (12) does not hold; i.e., this series has sum infinity. Define the functions $f_{n}:[0,1) \rightarrow R$ by

$$
f_{n}(x)=\frac{\left|S_{n}(x)\right|^{2}}{\sum_{i=1}^{n}\left|b_{i}\right|^{2}} \text { for } n=1,2, \ldots
$$

Using (8) we get for any $n \in \mathbb{N} \int f_{n}(x) d x=1$.

Consider the function $f:[0,1) \rightarrow \mathbb{R}$ given by $f(x)=\varlimsup_{n \rightarrow \infty} f_{n}(x)$. We claim that $\int f(x) d x>0$. Assume to the contrary that $f=0$ almost everywhere. We will show that this leads to a contradiction. For any $0<\varepsilon<\frac{1}{3}$ and $n \in \mathbb{N}$ consider the set $A_{n}^{\varepsilon}=\left\{x ; f_{n}(x)>\varepsilon\right\}$. It follows that $\lambda\left(A_{n}^{\varepsilon}\right) \rightarrow 0$ as $n \rightarrow \infty$. Indeed, if this is not true, then the set $\bigcap_{n=1}^{\infty} \bigcup_{k=n}^{\infty} A_{k}^{\varepsilon}$ would have positive measure. Also for any $x$ in this set $f(x)>0$ contrary to the fact that $f=0$ almost everywhere.

Find an $k \in \mathbb{N}$ for which we have $\lambda\left(A_{n}^{\varepsilon}\right)<\varepsilon$ for all $n \geq k$. Let $B_{n}^{\varepsilon}=$ $\left\{x ; f_{n}(x) \leq \varepsilon\right\}$ and $C_{n}=\left\{x ; f_{n}(x) \geq 1\right\}$. We have that $\lambda\left(B_{n}^{\varepsilon}\right)>1-\varepsilon$ and $\lambda\left(C_{n}\right)<\varepsilon$. Since $\int f_{n} d x=1$, we get that

$$
\int_{C_{n}} f_{n} d x=1-\int_{[0,1] \backslash\left(C_{n} \cup B_{n}^{\varepsilon}\right)} f_{n} d x-\int_{B_{n}^{\varepsilon}} f_{n} d x \geq 1-2 \varepsilon .
$$

Consequently $\int_{C_{n}}\left(f_{n}-1\right) d x \geq 1-3 \varepsilon$. Using Hölder's inequality we get

$$
1-3 \varepsilon \leq \int_{C_{n}}\left(f_{n}-1\right) d x \leq\left(\int_{C_{n}} 1 d x\right)^{1 / 2}\left(\int_{C_{n}}\left(f_{n}-1\right)^{2} d x\right)^{1 / 2} .
$$


From this $\int_{C_{n}}\left(f_{n}-1\right)^{2} d x \geq \frac{(1-3 \varepsilon)^{2}}{\varepsilon}$. If we let $\varepsilon \rightarrow 0+$, we conclude that

$$
\lim _{n \rightarrow \infty} \int\left(f_{n}-1\right)^{2} d x \geq \lim _{n \rightarrow \infty} \int_{C_{n}}\left(f_{n}-1\right)^{2} d x=\infty .
$$

On the other hand a direct computation gives us $\left(f_{n}-1\right)^{2}=\frac{4 K_{n}(x)^{2}}{\left(\sum_{i=1}^{n}\left|b_{i}\right|^{2}\right)^{2}}$, where $K_{n}(x)$ is defined as before. By (11) we get

$$
\begin{aligned}
\int\left(f_{n}-1\right)^{2} d x & =\frac{4 \sum_{1 \leq i<j \leq n}\left|\left\langle b_{i}, b_{j}\right\rangle\right|^{2}}{\left(\sum_{i=1}^{n}\left|b_{i}\right|^{2}\right)^{2}} \leq \frac{2 \sum_{1 \leq i \neq j \leq n}\left|b_{i}\right|^{2}\left|b_{j}\right|^{2}}{\left(\sum_{i=1}^{n}\left|b_{i}\right|^{2}\right)^{2}} \\
& \leq 2 \frac{\left(\sum_{i=1}^{n}\left|b_{i}\right|^{2}\right)^{2}}{\left(\sum_{i=1}^{n}\left|b_{i}\right|^{2}\right)^{2}}=2 .
\end{aligned}
$$

However, this contradicts (13). Therefore we cannot have $f=0$ almost everywhere.

Now we can continue our proof. Clearly, since $\int f d x>0$ and $f(x) \geq 0$ there is $\varepsilon>0$ such that $\lambda(A)>0$ where $A=\{x \in[0,1) ; f(x)>\varepsilon\}$. We claim is that the set

$$
B=\left\{x \in[0,1) ; \varlimsup_{n \rightarrow \infty}\left|S_{n}(x)\right|^{2}=\infty\right\}
$$

has measure one. To see this is not difficult. First of all we show that $A \subset B$ and therefore $B$ has positive measure. This and the obvious fact that the set $B$ is homogenous (by Proposition 3.3) give us (by Proposition 3.2) that the measure of the set $B$ must be one.

Pick any $x \in A$. Then there is an infinite increasing sequence of integers $\left(n_{i}\right)_{i \in \mathbb{N}}$ such that

$$
f_{n_{i}}(x)>\varepsilon \text {; i.e., }\left|S_{n_{i}}(x)\right|^{2}>\varepsilon \sum_{j=1}^{n_{i}}\left|b_{j}\right|^{2} \rightarrow \infty \text { as } i \rightarrow \infty \text {. }
$$

Therefore $x \in B$, so $A \subset B$ is established.

Finally, if $x \in \varphi(\mathcal{B})$, then $x \notin B$. The complement of $B$ has measure zero which is also the measure of the set $\varphi(\mathcal{B})$. Since $\mathcal{C} \subset \mathcal{B}$ also $\lambda(\varphi(\mathcal{C}))=0$. This finishes our proof.

Remark. [Remark about "almost" orthogonality] If we look at the second part of the proof of Theorem 3.8, we see that the measure of the set

$$
B=\left\{x \in[0,1) ; \varlimsup_{n \rightarrow \infty}\left|S_{n}(x)\right|^{2}=\infty\right\}
$$


is one. One might ask whether this claim cannot be improved; i.e., we would like to show that the measure of the set

$$
C=\left\{x \in[0,1) ; \lim _{n \rightarrow \infty}\left|S_{n}(x)\right|^{2}=\infty\right\}
$$

is one. Unfortunately this claim is not true. Let us take $H=\mathbb{R}$. We will show that the set

$$
D=\left\{x \in[0,1) ; \varlimsup_{n \rightarrow \infty} S_{n}(x)=\infty \text { and } \varliminf_{n \rightarrow \infty} S_{n}(x)=-\infty\right\}
$$

has measure one; i.e., almost everywhere the sequence of partial sums $\left(S_{n}\right)_{n \in \mathbb{N}}$ of the series (1) is oscillatory. Therefore if for example $\left|b_{n}\right| \leq K$; i.e. the sequence $\left(b_{n}\right)_{n \in \mathbb{N}}$ is bounded in absolute value by $K$ it follows that for almost any $x \in[0,1)$ and any $n \in \mathbb{N}$ there is $k \in \mathbb{N}, \quad k \geq n$ such that $\left|S_{k}(x)\right| \leq K$. Thus $x \notin C$.

To see why $D$ has measure one we write $B$ as

$$
B=\left\{x \in[0,1) ; \varlimsup_{n \rightarrow \infty} S_{n}(x)=\infty\right\} \cup\left\{x \in[0,1) ; \varliminf_{n \rightarrow \infty} S_{n}(x)=-\infty\right\} .
$$

Let us call the sets on the right side $B_{1}$ and $B_{2}$, respectively. Since the mapping $x \mapsto 1-x$ maps $B_{1}$ onto $B_{2}$ and vice versa, these sets have same measure. Their union set $B$ has measure one; i.e., $B_{1}$ and $B_{2}$ have measure at least $\frac{1}{2}$. Moreover they are homogenous and therefore their measure must be one. Now the set $D$ can be written as $B_{1} \cap B_{2}$ which means its measure is also one.

A different situation might happen if the space $H$ is infinite dimensional. We will call the elements $b_{1}, b_{2}, \ldots$ "almost" orthogonal if

$$
\sum_{1 \leq i<j}^{\infty}\left\langle b_{i}, b_{j}\right\rangle^{2}=K<\infty
$$

In this case by limiting $n \rightarrow \infty$ in the Lemma 3.7 we get

$$
P\left(\max _{i \in \mathbb{N}}\left|K_{i}\right| \geq \varepsilon\right) \leq \frac{1}{\varepsilon^{2}} \sum_{1 \leq i<j}^{\infty}\left|\left\langle b_{i}, b_{j}\right\rangle\right|^{2}=\frac{1}{\varepsilon^{2}} K .
$$

Take $\varepsilon=\sqrt{2 K}$. We get for the set $E=\left\{x \in[0,1) ; \max _{i \in \mathbb{N}}\left|K_{i}(x)\right| \geq \sqrt{2 K}\right\}$ that $P(E) \leq \frac{1}{2}$; i.e., $P\left(E^{c}\right)=P([0,1) \backslash E) \geq \frac{1}{2}$.

Now our claim is that the set $C$ has positive measure. In fact we have that $E^{c} \subset C$ (from (9)). For any $x \in E^{c}$ we get that

$$
\left|S_{n}\right|^{2}=\sum_{i=1}^{n}\left|b_{i}\right|^{2}+2 K_{n} \geq \sum_{i=1}^{n}\left|b_{i}\right|^{2}-2 \sqrt{2 K} \rightarrow \infty .
$$


So the measure of the set $C$ is positive. Again since $C$ is homogenous it must have measure one. One trivial example of "almost" orthogonal sequence $b_{1}, b_{2}, b_{3}, \ldots$ might be the sequence

$$
\begin{aligned}
& b_{1}=(1,0,0,0, \ldots) \in \ell^{2}, \\
& b_{2}=(0,1,0,0, \ldots) \in \ell^{2}, \\
& b_{3}=(0,0,1,0, \ldots) \in \ell^{2}, \text { etc. }
\end{aligned}
$$

In fact this sequence is orthogonal in $H=\ell^{2}$. Now the sum (14) is finite (equal to zero) and therefore the set $C$ has measure one; i.e. almost all $S_{n}(x)$ tends to infinity in norm. (For this trivial example one can show directly that for any $x:\left|S_{n}(x)\right|^{2}=n$ ) We formulate the observation above into the following proposition.

Proposition 3.10. If the elements $b_{1}, b_{2}, b_{3}, \cdots \in H$ are "almost" orthogonal, i.e., $\sum_{1 \leq i<j}^{\infty}\left\langle b_{i}, b_{j}\right\rangle^{2}<\infty$ and $\sum_{n=1}^{\infty}\left|b_{n}\right|^{2}=\infty$, then the set

$$
\left\{x \in[0,1) ; \lim _{n \rightarrow \infty}\left|\sum_{i=1}^{n}(-1)^{a_{i}(x)} b_{i}\right|=\infty\right\}
$$

has measure one.

We finish with a very nice example illustrating Theorem 3.8 in the case $H=R$ :

Example 3.11. Consider the series $\sum_{n=1}^{\infty}(-1)^{a_{n}} \frac{1}{n^{\beta}}$. For $\frac{1}{2}<\beta \leq 1$ we get that this series converges almost everywhere and for $0<\beta \leq \frac{1}{2}$ this series diverges almost everywhere.

\section{Porosity}

In this section we briefly discuss the question whether the Theorem 2.2 could strengthened by replacing words "of the first Baire category" by words "porous" or " $\sigma$-porous". In other words we ask whether the sets $\mathcal{C}$ and $\mathcal{B}$ are porous ( $\sigma$-porous) in the complete metric space $\left(\{0,1\}^{\mathbb{N}}, d_{E}\right)$.

The results from the Section 3 implies that in general the answer is no, since porosity or $\sigma$-porosity of the set $\mathcal{C}$ would imply porosity ( $\sigma$-porosity) of the set $\varphi(\mathcal{C})$ on the real interval $[0,1]$. But such set $\varphi(\mathcal{C})$ has Lebesgue measure zero. On the other hand we saw that there are many sequences $\left(b_{n}\right)_{n \in \mathbb{N}}$ for which corresponding set $\varphi(\mathcal{C})$ is of full Lebesgue measure. Nevertheless it is possible that there exists certain restrictive condition on the sequence $\left(b_{n}\right)_{n \in \mathbb{N}}$ for which 
we get desired porosity. Unfortunately, we were not able to find it, our best result (by following the proof of Theorem 2.2) yielded $\varlimsup_{n \rightarrow \infty}\left|b_{n}\right|>0$, which has questionable value, since for such a sequence the set $\mathcal{C}$ is empty. This might lead to a conjecture that in fact for any sequence $\left(b_{n}\right)_{n \in \mathbb{N}}$ for which $\lim _{n \rightarrow \infty} b_{n}=0$ the set $\mathcal{C}$ cannot be $\sigma$-porous. We were not able to prove this conjecture nor find any counterexample. Naturally, a similar question makes sense also for the set $\mathcal{B}$.

Acknowledgement The author wishes to thank the referee for a careful review of the paper, in particular for the detection of a couple of miscalculations and several misprints.

\section{References}

[AMM] H. D. Rudeman, Advanced problem 6105, American Math. Monthly, 83, (1976).

[B] P. Bunschuch, Konvergenz unendlicher Reihen und Gleeichverteillung mod 1, Arch. Math., (1977), 518-523.

[C] E. Cesàro, Sur une distribution de signes, Rend. Acc. Lincei, 4 (1888), 133.

[D1] M. Dindoš, On a typical series with alternating signs, Real. Anal. Exch., this issue.

[D2] M. Dindoš, On sign distribution in relatively convergent series, Preprint (Downloadable at the site http://www . math. unc.edu/Grads/dindos/publ.html), (1999).

[Du] Richard Durret Probability: Theory and Examples, Duxbury press, (1995).

[L-ک̌ $\quad$ V. László and T. Šalát, The structure of some sequence spaces, and uniform distribution (mod 1), Period. Math. Hung., 10, (1979), 8998.

[Š] T. Šalát, On subseries of divergent series, Matematický časopis, 18 (1968), 312-338.

[Z] V. Zanelli, Sign distribution and absolutely divergent series, Periodica Mathematica Hungarica, 28(1), (1994), 1-17. 